\title{
Metabolic flux responses to genetic modification for shikimic acid production by Bacillus subtilis strains
}

Dong-Feng Liu ${ }^{1,2}$, Guo-Min Ai ${ }^{1}$, Qing-Xiang Zheng ${ }^{3}$, Chang Liu' ${ }^{1}$ Cheng-Ying Jiang ${ }^{1}$, Li-Xia Liu ${ }^{3}$, Bo Zhang ${ }^{1}$, Yi-Ming Liu', Chen Yang ${ }^{3^{*}}$ and Shuang-Jiang Liu ${ }^{1,4^{*}}$

\begin{abstract}
Background: Shikimic acid $(S A)$ is a key chiral starting molecule for the synthesis of the neuramidase inhibitor GS4104 against viral influenza. Microbial production of SA has been extensively investigated in Escherichia coli, and to a less extent in Bacillus subtilis. However, metabolic flux of the high SA-producing strains has not been explored. In this study, we constructed with genetic manipulation and further determined metabolic flux with ${ }^{13} \mathrm{C}$-labeling test of high SA-producing B. subtilis strains.

Results: $B$. subtilis $1 \mathrm{~A} 474$ had a mutation in SA kinase gene (arol) and accumulated $1.5 \mathrm{~g} / \mathrm{L}$ of SA. Overexpression of plasmid-encoded aro $A$, aroB, aro $C$ or aroD in $B$. subtilis revealed that aroD had the most significantly positive effects on SA production. Simultaneous overexpression of genes for 3-deoxy-D-arabinoheptulosonate-7-phosphate synthase (aroA) and SA dehydrogenase (aroD) in B. subtilis BSSA/pSAAroA/pDGSAAroD resulted in SA production of $3.2 \mathrm{~g} / \mathrm{L}$. ${ }^{13} \mathrm{C}-$ Metabolic flux assay (MFA) on the two strains BSSA/pHCMC04/pDG148-stu and BSSA/pSAAroA/pDGSAAroD indicated the carbon flux from glucose to SA increased to $4.6 \%$ in BSSA/pSAAroA/pDGSAAroD from $1.9 \%$ in strain BSSA/pHCMC04/pDG148-stu. The carbon flux through tricarboxylic acid cycle significantly reduced, while responses of the pentose phosphate pathway and the glycolysis to high SA production were rather weak, in the strain BSSA/pSAAroA/pDGSAAroD. Based on the results from MFA, two potential targets for further optimization of SA production were identified. Experiments on genetic deletion of phosphoenoylpyruvate kinase gene confirmed its positive influence on SA production, while the overexpression of the transketolase gene did not lead to increase in SA production.
\end{abstract}

Conclusion: Of the genes involved in shikimate pathway in B. subtilis, aroD exerted most significant influence on SA accumulation. Overexpression of plasmid-encoded aroA and aroD doubled SA production than its parent strain. MFA revealed metabolic flux redistribution among phosphate pentose pathway, glycolysis, TCA cycle in the Iow and high SA-producing B. subtilis strains. The high SA producing strain BSSA/pSAAroA/pDGSAAroD had increased carbon flux into shikimate pathway and reduced flux into TCA cycle.

Keywords: Shikimic acid production, Shikimate pathway, Bacillus subtilis, Metabolic flux assay (MFA), aroA, aroD, tkt, pyk

\footnotetext{
* Correspondence: chenyang@sibs.ac.cn; liusj@im.ac.cn

${ }^{3}$ Key Laboratory of Synthetic Biology, Institute of Plant Physiology and Ecology, Shanghai Institute for Biological Sciences, Chinese Academy of Sciences, Shanghai 200032, China

'State Key Laboratory of Microbial Resources, Institute of Microbiology,

Chinese Academy of Sciences, Beijing 100101, China

Full list of author information is available at the end of the article
}

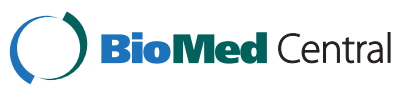

(C) 2014 Liu et al.; licensee BioMed Central Ltd. This is an Open Access article distributed under the terms of the Creative Commons Attribution License (http://creativecommons.org/licenses/by/2.0), which permits unrestricted use, distribution, and reproduction in any medium, provided the original work is properly credited. The Creative Commons Public Domain Dedication waiver (http://creativecommons.org/publicdomain/zero/1.0/) applies to the data made available in this article, unless otherwise stated. 


\section{Background}

Shikimic acid (3,4,5-trihydroxy-1-cyclohexene-1-carboxylic acid, SA), is a key chiral starting material for the synthesis of the antiviral neuraminidase inhibitor GS4104 [1,2]. It is a key metabolic intermediate of the shikimate pathway for biosynthesis of aromatic amino acids (L-Phe, L-Trp, and L-Tyr) and many alkaloids in plants and microorganisms [3-5]. As a commercial product, SA has been extracted from the fruits of the Illicium plant. However, microbial fermentation as an alternative process for SA production has attracted more and more interests.

Beginning with phosphoenolpyruvate (PEP) and erythrose-4-phosphate (E4P), SA is synthesized via first 4 reactions of the shikimate pathway (Figure 1). This shikimate pathway is essential to bacterial growth and there has been no report on excessive accumulation of SA by microorganisms. Genetic manipulation and metabolic engineering approaches had been applied to modify

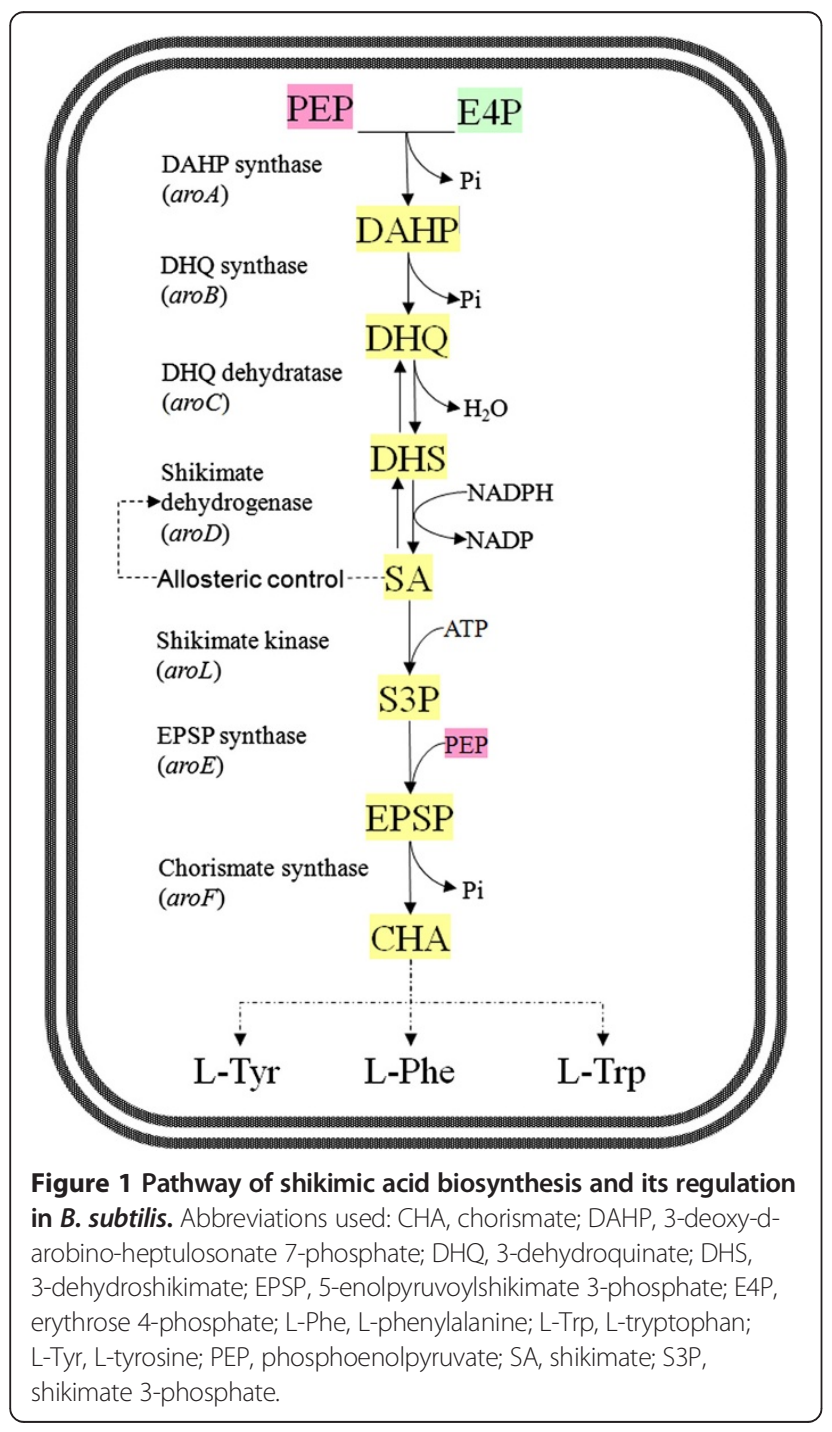

several microorganisms, particular Escherichia coli, for SA accumulation [6-9]. One strategy was to increase the supply of PEP and/or E4P, thus more carbon substrate channeled into SA synthesis. For examples, the PEPconsuming glucose transfer system (PTS) was replaced by non-PEP-consuming glucose facilitators, and transketolase I $(t k t A)$ and PEP synthase (ppsA) genes were overexpressed to increase the availability of E4P and PEP [10-14]. A second strategy was to block SA flux into chorismic acid by inactivating SA kinases (aroK and aroL) genes, and resulting the accumulation of SA in large quantity $[6,15]$. A third strategy was overexpression of feedback-resistant (fbr) 3-deoxy-D-arabinoheptulosonate7-phosphate (DAHP) synthase genes (e.g. arof $f^{f b r}$ and aro $\left.G^{f b r}\right)$, thus avoided feedback inhibition in the first step of the shikimate pathway $[7,16]$. It was reported that high amount of extracellular shikimic acids activated the shikimic acid transporter ShiA (shiA) and invoked transport of SA back into cells. So, a forth strategy was to inactivate the shiA thus vanished the uptake of SA back into cell and diminished intracellular accumulation of 3-dehydroshikimic acid as well [9]. Besides E. coli, efforts were also made to convert Bacillus subtilis into a SA accumulator. According to a patent description [17], genetic deletion of SA kinase gene (arol) and overexpression of bacilli DAHP synthase (aroA) and SA dehydrogenase $(a r o D)$ genes resulted in a significant accumulation of SA in B. subtilis. So far as we know, intensive evaluation of aro genes on SA production has not been reported in $B$. subtilis. Furthermore, knowledge of the metabolic fluxes of those genetically engineered strains of $E$. coli and $B$. subtilis is still missing.

In this study, we investigated the metabolic fluxes with ${ }^{13} \mathrm{C}$-labeled glucose in two genetically modified, SA-producing strains of $B$. subtilis, namely BSSA/pHCM C04/pDG148-stu and BSSA/pSAAroA/pDGSAAroD. Our results indicated that significant metabolic flux changes occurred for the shikimate pathway, tricarboxylic acid (TCA) cycle, and reactions involving PEP and E4P generation and consumption. Based on the ${ }^{13} \mathrm{C}$-labeling metabolic flux assay (MFA), we further created mutants for SA production. The highest SA-producing B. subtilis strain produced $3.46 \mathrm{~g} / \mathrm{L}$ of SA during batch cultivation in flasks.

\section{Results}

\section{Strain construction and SA productivity}

B. subtilis strain 1A474 had been used for genome mapping and its genome had a mutation on the SA kinase gene (aroI). In this study, we identified that this specific mutation of aroI was resulted from a single mutation of $\mathrm{C}$ to $\mathrm{G}$, leading to $\operatorname{Arg}^{129}$ (coded by CGC) being replaced by Cys $^{129}$ (UGC). Phenotypically, strain 1A474 was not able to grow on minimal medium and it restored growth 
when supplemented with L-Phe, L-Trp, and L-Tyr. We determined that strain $1 \mathrm{~A} 474$ accumulated up to $1.5 \mathrm{~g} / \mathrm{L}$ of SA in batch cultivation (Table 1).

Strain 1A474 was used to construct high SA producers. For this purpose, we adopted a strategy to overexpress aro genes involving shikimate pathway in strain 1A474. The gene $\operatorname{aro} A, \operatorname{aro} B, \operatorname{aro} C$, and $\operatorname{aro} D$ were cloned from the genome of strain 168 and were individually overexpressed in strain $1 \mathrm{~A} 474$. SA production by the resulting recombinant strains was determined and was compared to their parent strains. Results showed that overexpression of plasmid-encoded aro $A$ (strain BSSA/pSAAroA), aro $B$ (strain BSSA/pSAAroB), or aroC (strain BSSA/pSAAroC), exhibited no significant increase in SA production, however, the overexpression of plasmid-encoded aroD in strain BSSA/pSAAroD resulted in higher production of SA $(2.3 \mathrm{~g} / \mathrm{L}$, an increase of $53 \%)$, compared to its parent strain $(1.5 \mathrm{~g} / \mathrm{L})$ (Table 1$)$.

In order to improve SA production further, we cooverexpressed $\operatorname{aro} A$, aroB, or aro $C$ with $\operatorname{aroD}$ in $B$. subtilis. Results showed that co-overexpression of aro $A$ (strain BSSA/pSAAroA/pDGSAAroD), aroB (strain BSSA/pSAAro $B / \mathrm{pDGSA} A$ roD), or aroC (strain BSSA/pSAAroC/pDG $S A A r o D)$ with aroD indeed increased SA production compared to strain BSSA/pHCMC04/ pDGSAAroD and parent strain 1A474 (Table 1). We also found that double overexpression of $a r o D$ on two plasmids further increased SA production compared to single overexpression of $\operatorname{aroD}$ (Table 1). Strain BSSA/pSAAroA/pDGSAAroD that cooverexpressed $\operatorname{aro} A$ and $a r o D$ was the best SA producer, with SA production of $3.2 \mathrm{~g} / \mathrm{L}$ during batch cultivation fermentation. Compared to the parent strain 1A474, SA production was doubled in strain BSSA/pSAAroA/ pDGSAAroD (Table 1).

\section{Phenotypic characterization of growth, glucose consumption and metabolite production with strains BSSA474a and BSSA47407}

Figure 2 showed cell growth $\left(\mathrm{OD}_{600}\right)$, glucose consumption, SA production, and 3-dehydroshikimic acid production of strains BSSA/pHCMC04/pDG148-stu and BSSA/pSA AroA/pDGSAAroD in M9 minimal medium supplemented with 5 g/L glucose. Strain BSSA/pHCMC04/pDG148stu grew slightly faster than BSSA/pSAAroA/pDGSAAroD did, but they reached almost identical cell mass $\left(\mathrm{OD}_{600}\right)$ at the end of cultivation. The consumption of glucose was faster by BSSA/pHCMC04/pDG148-stu than BSSA/ pSAAroA/pDGSAAroD. The concentrations of two metabolites, namely SA and 3-dehydroshikimic acid, were determined. Strain BSSA/pHCMC04/pDG148-stu produced equal amounts of SA and 3-dehydroshikimic acid (Table 2, $1.1 \mathrm{mM}$ ), at a molar ratio of 1:1. Strain BSSA/ pSAAroA/pDGSAAroD accumulated more SA (1.49 mM) than 3-dehydroshikimic acid $(0.35 \mathrm{mM})$, at a molar ratio of 4.3:1. For the purpose of metabolic flux analysis, we calculated the specific cell growth $(\mu)$, specific glucose consumption $\left(\mathrm{q}_{\mathrm{glu}}\right)$, specific shikimic acid production $\left(\mathrm{q}_{\mathrm{sa}}\right)$, and specific 3-dehydroshikimic acid production $\left(\mathrm{q}_{\mathrm{dhs}}\right)$ rates, and they are listed in Table 2.

\section{Metabolic flux responses to high production of SA in strain BSSA47407 \\ Metabolic flux analysis by GC/LC-MS}

The ${ }^{13} \mathrm{C}$-based metabolic flux analysis was performed for the strains BSSA/pHCMC04/pDG148-stu and BSSA/ pSAAroA/pDGSAAroD grown in the medium containing either $100 \%\left[1-{ }^{13} \mathrm{C}\right]$ glucose or $20 \%\left[\mathrm{U}-{ }^{13} \mathrm{C}\right]$ glucose and $80 \%$ unlabeled glucose. The mass isotopomer patterns in

Table 1 Production of shikimic acid by various recombinant strains of Bacillus subtilis

\begin{tabular}{|c|c|c|c|}
\hline Strains & Shikimic acid*(g/L) & Changes (\%) & Notes $^{\#}$ \\
\hline $1 \mathrm{~A} 474$ & $1.50 \pm 0.22$ & 100.0 & Parent strain \\
\hline $\mathrm{BSSA} / \mathrm{pHCMCO4}$ & $1.81 \pm 0.18$ & 120.7 & Parent strain carrying pHCMCO4 \\
\hline BSSA/pSAAroA & $1.42 \pm 0.20$ & 94.7 & Overexpression of aroA \\
\hline BSSA/pSAAroB & $1.74 \pm 0.23$ & 116.0 & Overexpression of $a r o B$ \\
\hline BSSA/pSAAroC & $1.77 \pm 0.15$ & 118.0 & Overexpression of aroC \\
\hline BSSA/pSAAroD & $2.30 \pm 0.17$ & 153.3 & Overexpression of aroD \\
\hline BSSA/pSAAroA/pDGSAAroD & $3.20 \pm 0.07$ & 213.3 & Overexpression of aroD plus aroA \\
\hline BSSA/pSAAroB/pDGSAAroD & $2.99 \pm 0.05$ & 199.3 & Overexpression of aroD plus aroB \\
\hline BSSA/pSAAroC/pDGSAAroD & $2.90 \pm 0.03$ & 199.3 & Overexpression of aroD plus aroC \\
\hline BSSA/pSAAroD pDGSAAroD & $2.91 \pm 0.04$ & 194.0 & Overexpression of aroD on two plasmids \\
\hline $\mathrm{BSSA}(\Omega t k t:: p S A T k t)$ & $3.11 \pm 0.03$ & 207.3 & Overexpression of tkt in BSSA/pSAAroA/pDGSAAroD \\
\hline $\operatorname{BSSA}(\Omega p y k:: p S A P y k)$ & $3.46 \pm 0.04$ & 230.7 & Knockout of pyk in BSSA/pSAAroA/pDGSAAroD \\
\hline
\end{tabular}

*The shikimic acid concentrations in the culture supernatants were averages of the results from three parallel cultures and standard errors were provided for shikimic acid production.

"For detailed genotypes, see Table 3. 

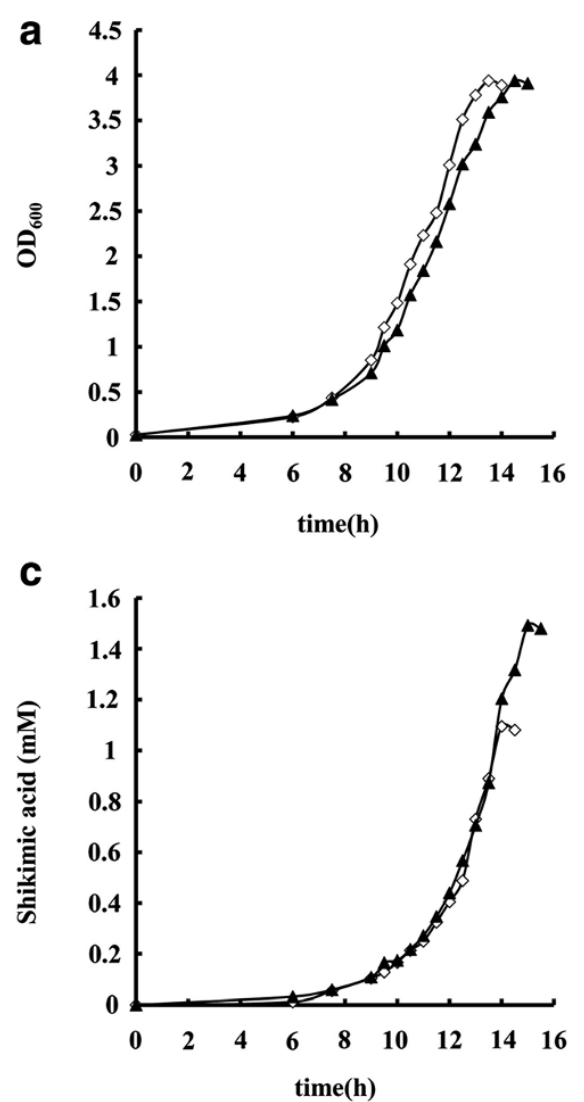
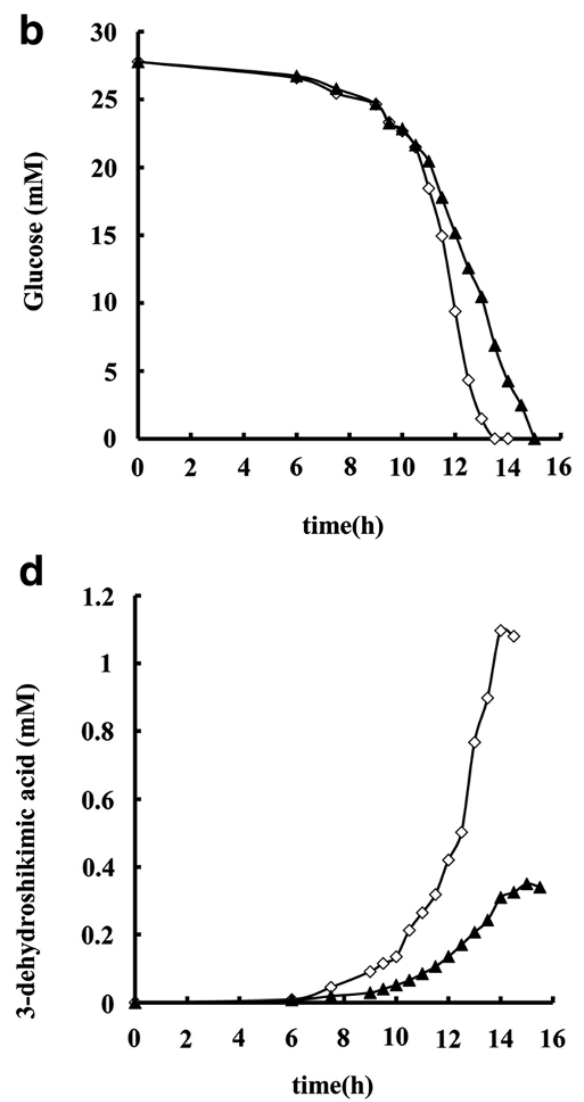

Figure 2 Time courses of cell growth (a), glucose consumption (b), shikimic acid production (c), and 3-dehydroshikimic acid production (d) of $B$. subtilis BSSA/pHCMC04/pDG148-stu $(0)$ and BSSA/pSAAroA/pDGSAAroD $(\mathbf{\Delta})$ in M9 minimal medium supplemented with $5 \mathrm{~g} / \mathrm{L}$ glucose.

cellular amino acids were analyzed by GC-MS (Additional file 1: Table S1). The intracellular phenylalanine and tyrosine were not labeled by ${ }^{13} \mathrm{C}$ because the SA kinase gene (aroI) was mutated in both strains and de nova synthesis of these amino acids from glucose was blocked. We measured the ${ }^{13} \mathrm{C}$ label state of extracellular shikimate by using LC-MS (Additional file 1: Table S1). To assess if the isotopic steady state was achieved, samples were taken at different time points during the exponential growth phase. The determined mass isotopomer distributions of key amino acids were almost unchanged with the time of harvest, which showed that a (quasi-) steady state could be reached during the exponential growth phase in batch cultures. From the GC-MS and LC-MS data (Additional file 1: Table S1), the ${ }^{13} \mathrm{C}$ labeling patterns of the precursor metabolites were identified, which allowed us to determine the metabolic flux ratios. The absolute net fluxes were then quantified by combining the flux ratios, physiological data (Table 1), and the biomass composition data [18]. The intracellular flux distribution in the parent strain BSSA/pHCMC04/pDG148-stu and high SA-producing strain BSSA/pSAAroA/pDGSAAroD are shown in Figure 3.

\section{Overall metabolic fluxes and responses of shikimate pathway}

We observed a significant flux redistribution of several metabolic reactions in responses to the overexpression of plasmid-encoded aro genes in the high SA-producing strain BSSA/pSAAroA/pDGSAAroD. The overall conversion of

Table 2 Determination of growth $\left(O D_{600}\right)$, specific cell growth rate $(\mu)$, specific glucose consumption rate ( $\left.q_{g l u}\right)$, specific shikimic acid production rate $\left(q_{\mathrm{sa}}\right)$, and specific 3-dehydroshikimic acid production rate $\left(q_{d h s}\right)$ of $B$. subtilis strains BSSA/pHCMC04/ pDG148-stu and BSSA/pSAAroA/pDGSAAroD

\begin{tabular}{|c|c|c|c|c|}
\hline Strains & $\mu\left(h^{-1}\right)$ & $q_{g l u}\left(m m o l ~ g^{-1} h^{-1}\right)$ & $\mathrm{q}_{\mathrm{sa}}\left(\mathrm{mmol} \mathrm{g} \mathrm{g}^{-1} \mathrm{~h}^{-1}\right)$ & $q_{d h s}\left(\mathrm{mmol} \mathrm{g}^{-1} \mathrm{~h}^{-1}\right)$ \\
\hline BSSA/pHCMC04/ pDG148-stu & $0.434 \pm 0.023$ & $5.199 \pm 0.120$ & $0.101 \pm 0.011$ & $0.132 \pm 0.013$ \\
\hline BSSA/pSAAroA/pDGSAAroD & $0.332 \pm 0.015$ & $4.666 \pm 0.132$ & $0.159 \pm 0.014$ & $0.048 \pm 0.007$ \\
\hline
\end{tabular}

Data are from 3 parallel cultivations and standard deviations are provided. B. subtilis strains were cultivaeted $250-\mathrm{mL}$ flasks that contained $50 \mathrm{~mL}$ of $\mathrm{M} 9 \mathrm{minimal}$ medium, which was supplemented to $5 \mathrm{~g} / \mathrm{L}$ glucose. Temperature and the shaking rate of the incubator were held constantly at $37^{\circ} \mathrm{C}$ and 200 rpm, respectively. 

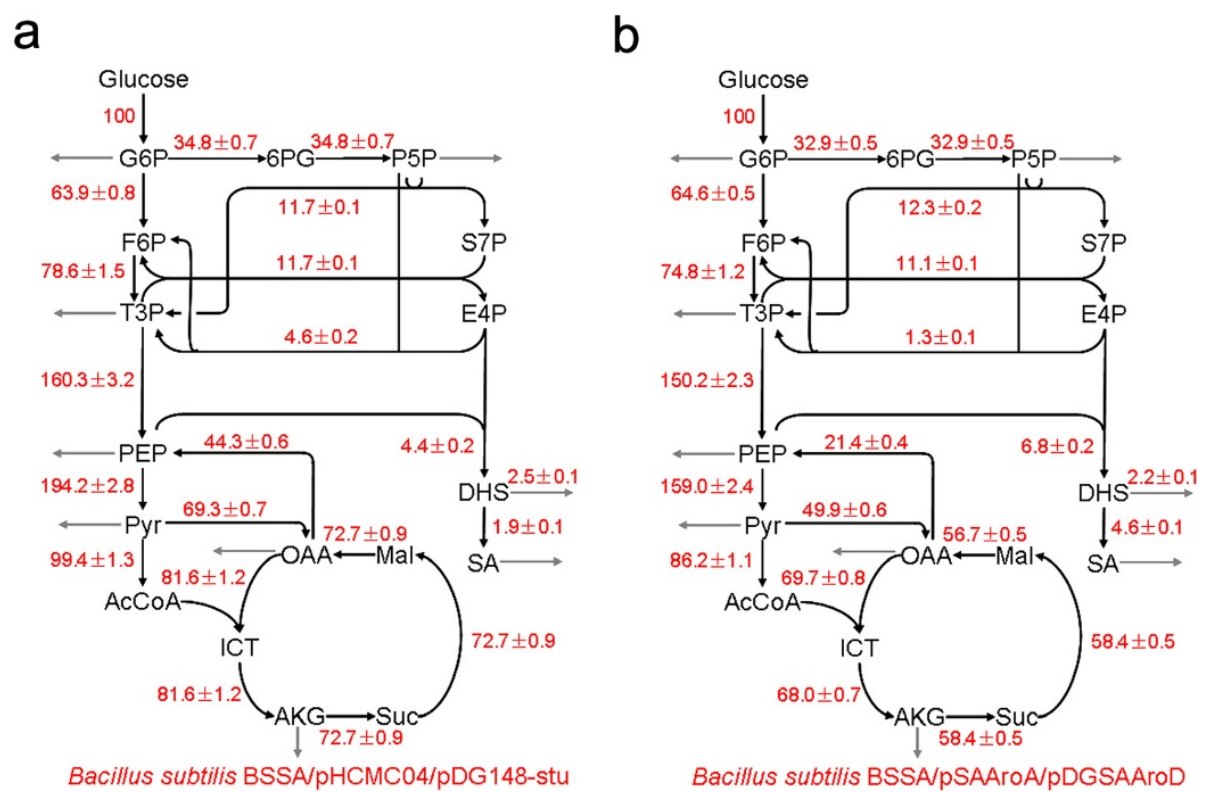

Figure 3 Metabolic flux distribution in B. subtilis BSSA/pHCMC04/pDG148-stu (a) and BSSA/pSAAroA/pDGSAAroD (b) during exponential growth phase. The estimated net fluxes were percentages of the relative rates normalized to the glucose uptake rates. Directions of net fluxes were represented by arrows. The gray arrows indicated flux related to biomass formation and fermentation products. The flux distributions were obtained from the best fit to the quantitative physiological data and the constraints derived from the MS measurements. F6P, fructose-6-phosphate; GAP, Glyceraldehyde 3-phosphate; AcCoA, acetyl-CoA; ICT, isocitrate; AKG, a-ketoglutarate; S7P, sedoheptulose-7-phosphate; DHS, 3-dehydroshikimic acid; SA, shikimic acid.

glucose into shikimate pathway increased to $6.8 \%$ in strain BSSA/pSAAroA/pDGSAAroD from $4.4 \%$ in strain BSSA/ pHCMC04/pDG148-stu. Consequently, the metabolic flux to SA increased from $1.9 \%$ in strain $\mathrm{BSSA} / \mathrm{pHCM}$ C04/pDG148-stu to $4.6 \%$ in strain BSSA/pSAAroA/ pDGSAAroD. We observed that the production rate of 3-dehydroshikimic acid decreased to $0.048 \mathrm{mmol} \mathrm{g}^{-1} \mathrm{~h}^{-1}$ in strain BSSA/pSAAroA/pDGSAAroD from $0.132 \mathrm{mmol}$ $\mathrm{g}^{-1} \mathrm{~h}^{-1}$ in the parent strain BSSA/pHCMC04/pDG148-stu (Table 2). Correspondingly, the metabolic flux from E4P and PEP into DHS decreased from $2.5 \%$ in the parent strain BSSA/pSAAroA/pDGSAAroD to $2.2 \%$ in strain $\mathrm{BSSA} / \mathrm{pSA} A$ roA/pDGSAAroD. The reversible reaction converting DHS to SA and being catalyzed by SA dehydrogenase (AroD) increased its flux by 2.4 folds (from $1.9 \%$ in BSSA/pHCMC04/pDG148-stu to $4.6 \%$ in BSSA/pSAAroA/ pDGSAAroD) in strain BSSA47407 (Figure 3).

\section{Flux shifts of TCA cycle, EMP and PPP pathways}

As shown in Figure 3, the flux through the TCA cycle was $81.6 \%$ relative to the glucose uptake rate in BSSA/ pHCMC04/pDG148-stu, while this flux decreased to $69.7 \%$ in BSSA/pSAAroA/pDGSAAroD. The overall flux of TCA cycle reduced significantly in the high SAproducing strain BSSA/pSAAroA/pDGSAAroD, suggesting a weak TCA cycle during high production of SA with $B$. subtilis. In contrast, fluxes of the EMP and pentose phosphate pathways did not shift significantly, except the reactions involving E4P generation and consumption. In the PPP pathway, transketolase, encoded by $t k t$, catalyzes reversible reaction (see Additional file 1: Appendix S1) and is that to E4P flux. We did not observe significant changes of flux driven by the transaldolase $\left(v_{6}\right)$. However, the net flux of reaction encoded by $t k t$ decreased from $4.6 \%$ in the parent strain $\mathrm{BSSA} / \mathrm{pHCMC04/pDG148-stu} \mathrm{to} 1.3 \%$ in the high SAproducing strain BSSA/pSAAroA/pDGSAAroD, suggesting that this reaction was severely disturbed and might be interesting to see how the SA synthesis would be influenced if $t k t$ were overexpressed.

\section{Supply of PEP for SA synthesis}

PEP is one of the initial substrates for shikimate synthesis. In almost all known microorganisms, the DAHP synthase catalyzes the condensation of PEP and E4P, which is the first reaction of the shikimate pathway. It is reasonable to consider any changes of PEP generation and consumption in the high SA-producing BSSA/pSAAroA/pDGSAAroD. Two reactions were considered in this study for generation of PEP: 1) The enolase (NP_391270.1, EC:4.2.1.11) catalyzes glycerate-2-phosphate into PEP $\left(v_{8}\right.$, see Additional file 1: Appendix S1). 2) The PEP-forming ATP:oxaloacetate carboxylyase (also named PEP carboxykinase, PckA, NP_39 0934.2, EC:4.1.1.49) catalyzes the conversion of oxaloacetate into PEP ( $v_{10}$, see Additional file 1: Appendix S1). As shown in Figure 3, flux into reaction $v_{8}$ decreased from $160.3 \%$ in BSSA/pHCMC04/pDG148-stu to $150.2 \%$ in BSSA/pSAAro $A /$ pDGSAAroD, and flux into reaction $v_{10}$ reduced from 
44.3\% in BSSA/pHCMC04/pDG148-stu to $21.4 \%$ in BSSA/ pSAAroA/pDGSAAroD. Meanwhile, we also observed that the conversion of PEP into pyruvate, which is encoded by pyk, also decreased, from 194.2\% in BSSA/pHCMC04/ pDG148-stu to $159.0 \%$ in BSSA/pSAAroA/pDGSAAroD. Taking all these flux information, we considered that the supply of PEP might in shortage in the high SA-producing BSSA/pSAAroA/pDGSAAroD. In addition, we observed that the flux of reaction $\left(v_{9}\right)$ in the SA-producing BSSA/ pSAAroA/pDGSAAroD was still high (159\%). For the purpose to increase supply of PEP to shikimate synthesis, knockout of this pyk might be a choice to increase SA production.

\section{Validation of MFA results: effects of tkt overexpression and pyk deletion on SA production}

Based on the previous results, we selected two targets for further genetic manipulation and to improve the SA production in B. subtilis. For the purpose to increase carbon flux to PEP, efforts were made to overexpress $t k t$ in BSSA/pSAAroA/pDGSAAroD. However, the resulting strain BSSA/ $\Omega$ tkt::pSAtkt did not show significant changes in SA production (Table 1). Further efforts were make to knock out the pyruvate kinase thus would diminish the flux of PEP into pyruvate. The resulting strain BSSA/ $\Omega$ pyk:: pSApyk produced $3.46 \mathrm{~g} / \mathrm{L}$ of SA, an increase of $8 \%$ compared to its parent strain BSSA/pSAAroA/pDGSAAroD.

\section{Discussion}

In this study, we investigated the influence of overexpression of aro genes on the accumulation of SA, and we constructed high SA producers by overexpression of aroA and $a r o D$ in $B$. subtilis. Our results demonstrated that overexpression of plasmid-encoded $a r o D$ increased the accumulation of SA, suggesting that AroD-driven reduction of 3-dehydroshikimate into SA was rate-limiting step for SA accumulation in B. subtilis. This is different from that of $E$. coli, of which the condensation of PEP and E4P into DAHP is the rate-limiting reaction and this reaction is subjected to extensive feedback regulations [19-21].

${ }^{13} \mathrm{C}$-MFA revealed carbon flux redistributions into shikimate pathway and TCA cycle, and reactions involving generation and consumption of PEP and E4P in genetically modified B. subtilis for SA production. Based on the MFA results in this study and previous investigation on E. coli $[7,8,13]$, $t k t$ and pyk were selected to be targets for genetically modification and for improvement of SA production with $B$. subtilis. Previous investigations showed that transformation with plasmid carrying $t k t A$ that encodes transketolase I increased the availability of E4P, and therefore increased SA and aromatic amino acid productions in E. coli [13]. However, this study showed that overexpression of $t k t$ did not increased SA production in B. subtilis, a remarkable difference from that in E. coli.
Since transketolase catalyzes a reversible reaction (see Additional file 1: Appendix S1), i.e., it generates E4P in one direction but consumes E4P in the other direction, we deduced that the $t k t$-encoded transketolase in B. subtilis catalyzed reaction more favored to direction of fructose 6-phosphate and glyceraldehyde 3-phosphate, and that the $t k t$-encoded transketolase in $E$. coli more favored the formation of E4P and xylulose 5-phosphate. Confirmation of this deduction needs further biochemical evidences on catalytic properties of the transketolases from B. subtilis and $E$. coli. These results suggest that MFA data need to be carefully interpreted and conclusions from MFA need to be experimentally confirmed, and application of MFA data from one bacterial species may be different from that of another bacterial species.

Based on MFA results as well as previous investigations in E. coli [7], a second target was spotted for knockout of pyk, so that consumption of PEP by this Pyk-driven reaction would be vanished. Pyruvate kinase, encoded by $p y k$, is an enzyme involved in glycolysis and catalyzes the consumption of one molecule of PEP, yielding one molecule of pyruvate and one molecule of ATP. Previous investigation revealed that PEP increased its intracellular pools when pyk was deleted [22]. We observed that SA production increased by $8 \%$ in B. subtilis, from $3.2 \mathrm{~g} / \mathrm{L}$ in the control strain BSSA/pSAAroA/pDGSAAroD to $3.46 \mathrm{~g} / \mathrm{L}$ in strain BSSA/ ppyk::pSApyk. This result is again different from that observed in E. coli pyk mutant, which did not showed obvious increase of intracellular PEP level [22]. Careful exploration of metabolic networks in $B$. subtilis and $E$. coli suggested that they have very different reactions at the PEP-pyruvate-oxaloacetate node, particularly the reactions for PEP and oxaloacetate generation/ consumption [23]. As demonstrated in previous studies, the pyk mutant of $B$. subtilis maintained high intracellular PEP levels [22] and produced less acetic acid than wild type did. This invoked the enthusiasm on exploitation of B. subtilis pyk mutants for production of various aromatic compounds. For example, B. subtilis strain BSTZ0402, an inducible pyk mutant, had been successfully constructed and yielded three folds higher folic acids compared to the parent strain B. subtilis 168 [24]. Additional works on mining the ${ }^{13} \mathrm{C}$-MFA data and exploitation of metabolic network from B. subtilis for improving SA productivity are in progress.

\section{Conclusion}

Based on the data obtained in this study, it was concluded that overexpression of plasmid-encoded aroD exerted most significant influence on SA accumulation in $B$. subtilis. Co-everexpression of plasmid-encoded aro $A$ and $a r o D$ doubled SA production than its parent strain BSSA/pHCMC04. So far as we know, this is the first time to quantify the flux flow in SA producing $B$. 
subtilis strains. MFA revealed metabolic flux redistribution among phosphate pentose pathway, glycolysis, TCA cycle in the low and high SA-producing B. subtilis strains. The high SA producing strain BSSA/pSAAroA/pDGSAAroD had increased carbon flux into shikimate pathway and reduced flux into TCA cycle. Based on the MFA results, $t k t$ and pyk were targeted for improving SA production by B. subtilis. Overexpression of plasmid-encoded $t k t$ did not increase SA production, and the deletion of $p y k$ increased SA production by $8 \%$ in B. subtilis. The results showed that B. subtilis and E. coli had very different responses to genetic manipulations of the aro genes involving shikimate pathway and the $t k t$ and pyk involving PEP/E4P generation and consumption.

\section{Materials and methods}

Microorganisms, plasmids, cultivation, and determination of cell growth

The bacterial strains, plasmids, and primers used in this study are listed in Table 3. B. subtilis strains 1A474 and 168 were obtained from the Bacillus Genetic Stock Center (BGSC) at the Ohio State University (http://www.bgsc. $\operatorname{org} /)$. Both B. subtilis and E. coli were routinely cultivated in Luria Bertani (LB) broth at $37^{\circ} \mathrm{C}$. When needed, antibiotics were included at the following concentrations, $15 \mu \mathrm{g} / \mathrm{mL}$ of erythromycin, chloramphenicol of $5 \mu \mathrm{g} / \mathrm{mL}$, ampicillin of $100 \mu \mathrm{g} / \mathrm{mL}$ and neomycin of $30 \mu \mathrm{g} / \mathrm{mL}$.

Cell growth was monitored by optical density measurements at $600 \mathrm{~nm}\left(\mathrm{OD}_{600}\right)$ using a UV/visible spectrophotometer. Cellular dry weights were determined with duplicate samples of culture broth, by precipitation of cells and washed with distilled water and lyophilization.

\section{Determination of SA and 3-dehydroshikimic acid by high performance liquid chromatography (HPLC)}

B. subtilis was cultivated in $\mathrm{LB}$ broth at $37^{\circ} \mathrm{C}$ and at $200 \mathrm{rpm}$, overnight, and cells from culture were used as inoculum. SA production was determined in triplicates after $90 \mathrm{~h}$ cultivation at $37^{\circ} \mathrm{C}$ and $200 \mathrm{rpm}$ in $250-\mathrm{mL}$ flasks that contained $50 \mathrm{~mL}$ of fermentation broth. The fermentation broth was modified from Iomantas et al. [17] by deletion of maltose, and had the following components (g/L): $\mathrm{K}_{2} \mathrm{HPO}_{4} \cdot 3 \mathrm{H}_{2} \mathrm{O}(18.3) ; \mathrm{KH}_{2} \mathrm{PO}_{4}$ (6); Urea (6); sucrose (80); $\mathrm{MgSO}_{4} \cdot 7 \mathrm{H}_{2} \mathrm{O}$ (1); $\mathrm{FeSO}_{4}(0.01) ; \mathrm{MnSO}_{4}$ (0.01); Yeast Extract (4). For induction of the target gene overexpression in recombinant $B$. subtilis strains, IPTG at final concentration of $0.05 \mathrm{mM}$ and xylose at final concentration of $5 \mathrm{~g} / \mathrm{L}$ were added into the fermentation broth.

SA and 3-dehydroskimic acid accumulation in culture supernatant was determined with an HPLC system (Agilent 1200 series) equipped with a SB C18 column $(4.6 \mathrm{~mm} \times$ $250 \mathrm{~mm} \times 5 \mu \mathrm{m})$. Five $\mathrm{ml}$ samples of fermentation broth were centrifuged at 13,000 rpm for $5 \mathrm{~min}$ on Microfuge ${ }^{\oplus} 18$ centrifuge (BECKMAN COULTER ${ }^{\mathrm{TM}}$ ) to remove cells, and the supernatant was applied for HPLC analysis. The HPLC was run with mixture of solution $\mathrm{A}$ (phosphoric acid in water, $\mathrm{pH}$ 2.5) and solution $\mathrm{B}$ (methanol) as eluant and was operated at a flow rate of $0.35 \mathrm{~mL} / \mathrm{min}$. SA and 3dehydroshikimic acid were detected at $215 \mathrm{~nm}$ with adiode array detector. The following gradient was used: at 0-7.5 min, 95\% of solution A and 5\% of solution B; at 7.5$8.0 \mathrm{~min}, 100 \%$ of solution B; at $8.0-15 \mathrm{~min}, 100 \%$ of solution B; $15.0-15.5 \mathrm{~min}, 95 \%$ of solution A and $5 \%$ of solution B; 15.5-22.5 min, $95 \%$ of solution A and $5 \%$ of solution B. Standard SA and 3-hydroskimic acid were eluted at 5.411 and $6.241 \mathrm{~min}$, respectively, under these conditions.

\section{Determination of glucose concentrations}

Glucose concentrations were enzymatically determined with glucose oxidase kit by using a bioanalyzer, according to producer's description (SBA-40C, Shandong Academy of Sciences).

\section{DNA extraction, amplification, plasmid construction and genetic transformation}

Plasmid and chromosomal DNAs were isolated using the Plasmid Minspin HP Kit and the SiMax ${ }^{\text {TM }}$ Genome DNA Kit, respectively. Purification of DNA fragments from agarose gels was done with the TIANgel Midi Purification Kit. Restriction enzymes, ligases and other DNA-manipulating enzymes were used according to their manufacturer's instructions.

The aro genes involving shikimate pathway, i.e., aro $A$ (Genbank accession number, NP_390853.1), aroB (NP_3 90151.1), aroC (NP_390189.1), and aroD (NP_390444.2) were PCR amplified from genomic DNA of B. subtilis strain 168 using primers listed in Table 3. The PCR products were digested with SpeI and XmaI and then cloned into the same digested pHCMC04 [25]. All plasmids (Table 3) constructed in this study were verified by DNA sequencing. Transformation of chemically competent cells of $E$. coli DH5 and of B. subtilis were carried out according to the manufacturer's manual or by the method of Spizizen [26]. In order to simultaneous overexpression of aro $A$ and $a r o D$ in $B$. subtilis, aroD was cloned into the pDG148-Stu vector [27], and the resulted plasmid was named pDGSAAroD (Table 3). Expression of the cloned aro genes in B. subtilis was induced by either $5 \mathrm{~g} / \mathrm{L}$ of xylose for pHCMC04 derivatives or $0.05 \mathrm{mM}$ IPTG for pDG148-Stu derivative.

Cloning and overexpression of $t k t$ (NP_389672.10) in B. subtilis were performed with the plasmid pMUTIN4 [28]. The $t k t$ with its ribosome binding site (RBS) was amplified by using primers listed in Table 3 and was cloned into the BamHI-XmaI treated backbone of pMUTIN4. Transformation of E. coli DH5 $\alpha$ and B. subtilis BSSA/ pSAAroA/pDGSAAroD and selection of transformants were performed as previously described. The B. subtilis 
Table 3 Bacillus subtilis strains, plasmids, and primers used in this study

\begin{tabular}{|c|c|c|}
\hline Strains/plasmids/primers & Relevant characteristics & Source/notes \\
\hline \multicolumn{3}{|l|}{ Strains } \\
\hline 168 & $\operatorname{trp} C 2$ & BGSC \\
\hline $1 \mathrm{~A} 474$ & amyE3 arol10 & BGSC \\
\hline $\mathrm{BSSA} / \mathrm{pHCMC04}$ & 1A474 carrying pHCMCO4 & This work \\
\hline BSSA/pSAAroA & 1A474 carrying pSAAroA & This work \\
\hline BSSA/pSAAroB & 1A474 carrying pSAAroB & This work \\
\hline BSSA/pSAAroC & 1A474 carrying pSAAroC & This work \\
\hline BSSA/pSAAroD & 1A474 carrying pSAAroD & This work \\
\hline BSSA/pHCMC04/pDG148-stu & 1A474 carrying pHCMCO4 and pDG148-stu & This work \\
\hline BSSA/pHCMC04/pDGSAAroD & 1A474 carrying pHCMCO4 and pDGSAAroD & This work \\
\hline BSSA/pSAAroA/ pDGSAAroD & 1 A474 carrying pSAAroA and pDGSAAroD & This work \\
\hline BSSA/pSAAroB pDGSAAroD & 1 A474 carrying pSAAroB and pDGSAAroD & This work \\
\hline BSSA/pSAAroC pDGSAAroD & 1 A474 carrying pSAAroC and pDGSAAroD & This work \\
\hline BSSA/pSAAroD pDGSAAroD & $1 \mathrm{~A} 474$ carrying $\mathrm{pSAAroD}$ and pDGSAAroD & This work \\
\hline 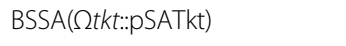 & BSSA/pSAAroA/pDGSAAroD harboring $\Omega$ tkt::pSATKT & This work \\
\hline 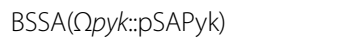 & BSSA/pSAAroA/pDGSAAroD harboring $\Omega p y k:: p S A P Y K$ & This work \\
\hline \multicolumn{3}{|l|}{ Plasmids } \\
\hline pHCMCO4 & bla $P_{x y \mid A} x y / R$ cat & BGSC \\
\hline pDG148-stu & neo ble Pspac lacl bla & BGSC \\
\hline PMUTIN4 & bla Pspac lacz lacl ermC & BGSC \\
\hline pSAAroA & pHCMCO4 derivative, aroA expressed from $\mathrm{P}_{x y / \mathrm{A}}$ & This work \\
\hline pSAAroB & pHCMC04 derivative, aro $B$ expressed from $P_{x y / A}$ & This work \\
\hline pSAAroC & pHCMCO4 derivative, aro $C$ expressed from $P_{x y / A}$ & This work \\
\hline pSAAroD & pHCMC04 derivative, aroD expressed from $\mathrm{P}_{x y / \mathrm{A}}$ & This work \\
\hline pDGSAroD & pDG148-stu derivative, aroD expressed from Pspac & This work \\
\hline pSATkt & bla Pspac-tkt-lacZ lacl ermC & This work \\
\hline pSAPyk & bla Pspac-pyk'-lacZ lacl ermC & This work \\
\hline \multicolumn{3}{|l|}{ Primers } \\
\hline aroA-fw-Spel & TCAACTAGTATGAGCAACACAGAGTTAG & Cloning of aroA into pHCMCO4 \\
\hline $\operatorname{aroA-rv-Xmal}$ & TCACCCGGGTTAAGCGTTGACTITCAC & \\
\hline aroB-fw-Spel & TCCACTAGTATGAAGACACTGCATGTTC & Cloning of aroB into $\mathrm{pHCMCO4}$ \\
\hline $\operatorname{aroB}-\mathrm{rv}-X m a l$ & TCAGGATCCTCATGATGTCTCCTCCAATC & \\
\hline aroc-fw-Spel & TCAACTAGTGTGAACGTGTTAACGATTAAAG & Cloning of aroC into pHCMCO4 \\
\hline aroc-rv-Xmal & TCACCCGGGCTATCCCCGTGTGTTITTATG & \\
\hline aroD-fw-Spel & TCCACTAGTATGAAAAAGCTGTACGG & Cloningof aroD into pHCMCO4 \\
\hline aroD-rv-Xmal & TCAGGATCCTTAACATTCTGTTCCTCC & \\
\hline aroD-DG-fw & AAGGAGGAAGCAGGTATGAAAAAGCTGTACGG & Cloning of aroD into pDG148-stu \\
\hline aroD-DG-rv & GACACGCACGAGGTTTAACATTCTGTTCCTCC & \\
\hline tkt-Mu-fw-Xmal & ACTCCCGGGACATAAGGAAGGGGATTITTATG & Cloning of tkt into pMUTIN4 \\
\hline tkt-Mu-rv-BamHI & ACTGGATCCACTCATCCTCTITCAAAAGC & \\
\hline tkt-ck-fw & TITCAGGAATACATAGAG & Confirming the integration of PSATKT \\
\hline tkt-ck-rv & TTAGACAAAATTTCTTC & \\
\hline pyk-Mu-fw-Xmal & CCCCCGGGGAAGATTTCAGAAGGAAGTGAACC & Cloning $5^{\prime}$ end of pyk into pMUTIN4 \\
\hline
\end{tabular}


Table 3 Bacillus subtilis strains, plasmids, and primers used in this study (Continued)

\begin{tabular}{lll}
\hline pyk-Mu-rv-BamHI & CCGGATCCGGAATTCCACACCTAAGTCTCC & Confirming the integration of pSAPYK \\
pyk-ck-fw & AACGTACATATGTAATCG & \\
pyk-ck-rv & CCGTTCAGCAAAAATAAAAC & \\
\hline
\end{tabular}

BGSC: Bacillus Genetic Stock Center. Resistance gene abbreviations as follows: bla, ampicillin; ermC, erythromycin and lincomycin; cat, chloramphenicol; neo, kanamycin. Other abbreviation: $\Omega$, insertion of integrated plasmid. Restriction sites were underlined.

transformants were selected on LB plates containing $15 \mathrm{ug} / \mathrm{mL}$ erythromycin, $5 \mathrm{ug} / \mathrm{mL}$ chloramphenicol, 30 $\mathrm{ug} / \mathrm{mL}$ neomycin and then analyzed by PCR to ensure the integration of a single copy of the plasmid into the target gene on the chromosome.

\section{Construction of an inducible pyk mutant}

The plasmid pMUTIN4 was also used to produce knockout mutations [28]. The fragment of 5' end of pyk (NP_3 90796.1) with its RBS was amplified from Bacillus subtilis 168 chromosomal DNA using primers listed in Table 3, and was cloned into the BamHI-XmaI treated backbone of pMUTIN4. Transformation of E. coli DH5 $\alpha$ and $B$. subtilis BSSA/pSAAroA/pDGSAAroD were performed as previously described. The $B$. subtilis transformants were selected on LB plates containing $15 \mu \mathrm{g} / \mathrm{mL}$ erythromycin, $5 \mu \mathrm{g} / \mathrm{mL}$ chloramphenicol, $30 \mu \mathrm{g} / \mathrm{mL}$ neomycin and $1 \mathrm{mM}$ IPTG and then analyzed by PCR to ensure that the $p y k$ gene had been knocked out.

\section{Growth and cultivation of $B$. subtilis with \\ ${ }^{13} \mathrm{C}$-labeled glucose}

The chemically defined M9 minimal medium [29] was applied for the ${ }^{13} \mathrm{C}$ labeling experiment. Glucose was added to M9 minimal medium as either entirely the $\left[1-{ }^{13} \mathrm{C}\right]-$ labeled isotope isomer (99\%; Cambridge Isotope Laboratories Inc., Andover, MA) or as a mixture of $20 \%(\mathrm{w} / \mathrm{w})$ $\left[\mathrm{U}-{ }^{13} \mathrm{C}\right]$ (99\%; Cambridge Isotope Laboratories Inc.) and $80 \%(\mathrm{w} / \mathrm{w})$ unlabeled glucose. B. subtilis strains pre-cultivated overnight in $5 \mathrm{~mL}$ of M9 minimal medium supplemented with $5 \mathrm{~g} / \mathrm{L}$ unlabeled glucose, and the precultures $(2 \mathrm{~mL})$ were used as inoculum for ${ }^{13} \mathrm{C}$-labeling experiments. The ${ }^{13} \mathrm{C}$-labeling experiments were performed in a $250-\mathrm{mL}$ flask that contained $50 \mathrm{~mL}$ of $\mathrm{M} 9$ minimal medium, which was supplemented with either 20:80 (w/w) mixture of $\left[\mathrm{U}^{13} \mathrm{C}\right]$ glucose:unlabeled glucose or $100 \%$ $\left[1-{ }^{13} \mathrm{C}\right]$ glucose. Temperature and the shaking rate of the incubator were held constant at $37^{\circ} \mathrm{C}$ and $200 \mathrm{rpm}$, respectively. When cells reached exponential phase, aliquots of $0.5 \mathrm{~mL}$ was aseptically collected from the culture at intervals of $0.5 \mathrm{~h}$. Measure the $\mathrm{OD}_{600}$ on a spectrometer to monitor the growth of the cells. For extracellular metabolite (shikimic acid and 3-dehydroshikimic acid) analysis by HPLC, culture samples were centrifuged for $5 \mathrm{~min}$ at $4^{\circ} \mathrm{C}$ and $20,000 \mathrm{~g}$ to remove the cells. The specific growth rate $(\mu)$ was defined as the increase in cell biomass concentration per unit time. The specific glucose consumption rate $\left(\mathrm{q}_{\mathrm{glu}}\right)$ was calculated during the exponential growth phase as the differential change in glucose with time $(t)$ normalized to the biomass concentration. The specific SA production rate $\left(\mathrm{q}_{\mathrm{sa}}\right)$, and the specific 3-dehydroshikicmic acid production rate $\left(\mathrm{q}_{\mathrm{dhs}}\right)$ were calculated during the exponential growth phase as the differential change in SA, and 3-dehydroshikicmic acid with time ( $t$ ) normalized to the biomass concentration, respectively.

\section{Preparation of cellular hydrolysate from ${ }^{13} \mathrm{C}$-labeled} biomass and measurements of ${ }^{13} \mathrm{C}$-labeled amino acids with gas chromatography and mass spectroscopy (GC-MS) Preparation of cellular hydrolysate from ${ }^{13} \mathrm{C}$-labeled biomass for the GC-MS measurement followed standard protocols [30]. For GC-MS measurement, the amino acids were derivatized with TBDMS and 1\% tert-butyldimethylchlorosilane at $85^{\circ} \mathrm{C}$ for $60 \mathrm{~min}$. The amounts of biomass used for GC-MS measurement was approximately $10 \mathrm{mg}$ of cellular dry weight. GC-MS was carried out using an Agilent GC-7890A gas chromatograph equipped with an Agilent HP-5MS column $(30 \mathrm{~m} \times 0.25 \mathrm{~mm} \times 0.25 \mathrm{~mm})$ that was directly connected to an MS-5975C mass spectrometer (Agilent). The following amino acids were detected: Ala, Asp, Glu, Gly, His, Ile, Leu, Lys, Met, Phe, Pro, Ser, Thr, Tyr, and Val.

\section{Determination of ${ }^{13} \mathrm{C}$-labeled SA and 3-dehydroshikimic acid with LC-MS}

Agilent 1290 UHPLC system equipped with ZORBAX 300SB-C18 Column $(2.1 \mathrm{~mm} \times 100 \mathrm{~mm} \times 1.8 \mu \mathrm{m})$ and Agilent 6530 Q-TOF mass spectrometer fitted with an electrospray ionization (ESI) source were used to measure ${ }^{13} \mathrm{C}$-labeling states of SA and 3-dehydroshikimic acid. SA and 3-dehydroshikimic acid was eluted with mixture of $0.1 \%$ formic acid in water (solution C) and acetonitrile (solution D) at ratio of 98:2 (solution C: solution D) and at a flow rate of $0.15 \mathrm{~mL} / \mathrm{min}$. MS data was collected in negative ion mode. The capillary voltage was set to $3500 \mathrm{kV}$, and the fragmentor voltage at $90 \mathrm{~V}$. The drying gas temperature was maintained at $350^{\circ} \mathrm{C}$ with a flow rate of $11 \mathrm{~L} / \mathrm{min}$ and a nebulizer pressure of $40 \mathrm{psi}$.

\section{Metabolic net-flux analysis}

${ }^{13} \mathrm{C}$ MFA was a well-established method, and details concerning the general modeling framework of classical 
stationary ${ }^{13} \mathrm{C}$ labeling experiments could be found elsewhere [30]. In the case of $\mathrm{U}^{13} \mathrm{C}$ labeling, several flux ratios, including (1) Pyr originating from malate, (2) PEP from OAA, (3) OAA from PEP, (4) PEP from transketolase, (5) PEP from the PP pathway, (6) pentose-5phosphate from Glu-6-P, (7) OAA from the glyoxylate shunt, (8) erythrose-4-phosphate from pentose-5-phosphate, (9) Ser from Gly, (10) Gly from Ser, and (11) PEP and E4P from shikimic acid were calculated from the $M D V_{\mathrm{M}}$ of precursors (Additional file 1: Table S2) with probabilistic equations. The calculating procedures were based on the general principle of flux ratio analysis [30-32] and were performed using a home-made Matlab program. Positional labeling pattern was also analyzed from cells grown exclusively on $\left[1-{ }^{13} \mathrm{C}\right]$ glucose to obtain precise information about the in vivo activities of the PPP and EMP pathways.

For quantification of carbon fluxes in B. subtilis, a bioreaction network was constructed. This network includes the reactions of the EMP, PP, and shikimate pathways, as well as the tricarboxylic acid (TCA) cycle and the glyoxylate shunt. The reactions catalyzed by PEP carboxykinase $\left(v_{10}\right)$, pyruvate carboxylase $\left(v_{11}\right)$, and malic enzyme $\left(v_{12}\right)$ were also included. The networks of active pathways identified by flux ratio analysis were used for flux quantification. The following enzyme reactions were considered reversible: phosphoglucose isomerase $\left(v_{2}\right)$, transketolase $\left(v_{5}\right.$ and $\left.v_{7}\right)$, transaldolase $\left(v_{6}\right)$, the sequence of glycolytic reactions leading from triose 3-phosphate to PEP $\left(v_{8}\right)$ and malate dehydrogenase $\left(v_{17}\right)$. The metabolic reactions considered as shown in the Additional file 1: Appendix S1.

All the above data, including (1) the stoichiometric reaction matrix, (2) the flux ratios derived from metabolic flux ratio analysis (Additional file 1: Table S3), (3) physiological data, and (4) precursor requirements for biomass synthesis, were utilized to calculate absolute fluxes in millimoles per gram of biomass per hour. Net fluxes were then estimated with Matlab by solving the stoichiometric matrix. The resulting flux distribution was the best fit to available data from both metabolite balances and tracer experiment-based flux ratios within the specified metabolic model.

\section{Statistical analysis}

Monte Carlo approach [33] was applied for statistical analysis.

\section{Additional file}

Additional file 1: Table S1. Mass isotopomer distribution of TBDMSderivatized protein-bound amino acids and shikimic acid (corrected). Table S2. Mass isotopomer distribution of precursors. Table S3. Flux ratio of Bacillus subtilis BSSA/pHCMC04/pDG148-stu and Bacillus subtilis BSSA/pSAAroA/ pDGSAAroD. Figure S1. ${ }^{13} \mathrm{C}$ fractional labeling (FL) of 15 proteinogenic amino acids and shikimic acid from Bacillus subtilis BSSA/pHCMC04/pDG148-stu (a) and Bacillus subtilis BSSA BSSA/pSAAroA/pDGSAAroD (b) during exponential growth phase. Appendix S1. Stoichiometric reactions implemented in the central metabolic network of Bacillus subtilis.

\section{Competing interests}

The authors declare that they have no competing interests.

\section{Authors' contributions}

SJL and CY conceived and designed the experiments. DFL and GMA performed the experiments. DFL, YML, QXZ, LXL, BZ, CL, CYJ, CY and SJL analyzed the data. DFL and $S J L$ wrote the manuscript. All authors read and approved the final manuscript.

\section{Acknowledgements}

This work was supported by 973 Project from Ministry of Science and Technology (No. 2012CB7211-04).

\section{Author details}

${ }^{1}$ State Key Laboratory of Microbial Resources, Institute of Microbiology, Chinese Academy of Sciences, Beijing 100101, China. ${ }^{2}$ School of Life Sciences, University of Science and Technology of China, Hefei 230026, China. ${ }^{3}$ Key Laboratory of Synthetic Biology, Institute of Plant Physiology and Ecology, Shanghai Institute for Biological Sciences, Chinese Academy of Sciences, Shanghai 200032, China. ${ }^{4}$ Institute of Microbiology, Chinese Academy of Sciences, Beichen-Xilu, Beijing, Chaoyang District 100101, China.

Received: 30 November 2013 Accepted: 21 February 2014

Published: 14 March 2014

\section{Reference}

1. Sheu TG, Deyde VM, Okomo-Adhiambo M, Garten RJ, Xu X, Bright RA, Butler EN, Wallis TR, Klimov Al, Gubareva LV: Surveillance for neuraminidase inhibitor resistance among human influenza $A$ and $B$ viruses circulating worldwide from 2004 to 2008. Antimicrob Agents Chemother 2008, 52:3284-3292.

2. Dharan NJ, Gubareva LV, Meyer JJ, Okomo-Adhiambo M, McClinton RC, Marshall SA, George KS, Epperson S, Brammer L, Klimov Al, Bresee JS, Fry AM: Infections with oseltamivir-resistant influenza $A(H 1 N 1)$ virus in the United States. Jama-j Am Med Assoc 2009, 301:1034-1041.

3. Bradley D: Star role for bacteria in controlling flu pandemic? Nat Rev Drug Discov 2005, 4:945-946.

4. Enrich LB, Scheuermann ML, Mohadjer A, Matthias KR, Eller CF, Newman MS, Fujinaka M, Poon T: Liquidambar styraciflua: a renewable source of shikimic acid. Tetrahedron Lett 2008, 49:2503-2505.

5. Lingens F: Biosynthesis of aromatic amino acids and its regulation. Angewandte Chemie-International Edition 1968, 7:350.

6. Kramer M, Bongaerts J, Bovenberg R, Kremer S, Muller U, Orf S, Wubbolts M, Raeven L: Metabolic engineering for microbial production of shikimic acid. Metab Eng 2003, 5:277-283.

7. Escalante A, Calderon R, Valdivia A, de Anda R, Hernandez G, Ramirez OT, Gosset G, Bolivar F: Metabolic engineering for the production of shikimic acid in an evolved Escherichia coli strain lacking the phosphoenolpyruvate: carbohydrate phosphotransferase system. Microb Cell Fact 2010, 9:21.

8. Dell KA, Frost JW: Identification and removal of impediments to biocatalytic synthesis of aromatics from D-glucose - rate-limiting enzymes in the common pathway of aromatic amino-acid biosynthesis. J Am Chem Soc 1993, 115:11581-11589.

9. Knop DR, Draths KM, Chandran SS, Barker JL, von Daeniken R, Weber W, Frost JW: Hydroaromatic equilibration during biosynthesis of shikimic acid. J Am Chem Soc 2001, 123:10173-10182.

10. Chandran SS, Yi J, Draths KM, von Daeniken R, Weber W, Frost JW: Phosphoenolpyruvate availability and the biosynthesis of shikimic acid. Biotechnol Prog 2003, 19:808-814

11. Yi J, Draths KM, Li K, Frost JW: Altered glucose transport and shikimate pathway product yields in E-coli. Biotechnol Prog 2003, 19:1450-1459.

12. Patnaik R, Liao JC: Engineering of Escherichia-Coli central metabolism for aromatic metabolite production with near theoretical yield. Appl Environ Microb 1994, 60:3903-3908. 
13. Patnaik R, Spitzer RG, Liao JC: Pathway engineering for production of aromatics in Escherichia-Coli - confirmation of stoichiometric analysis by independent modulation of Arog, Tkta, and Pps activities. Biotechnol Bioeng 1995, 46:361-370.

14. Lu JL, Liao JC: Metabolic engineering and control analysis for production of aromatics: role of transaldolase. Biotechnol Bioeng 1997, 53:132-138.

15. Draths KM, Knop DR, Frost JW: Shikimic acid and quinic acid: replacing isolation from plant sources with recombinant microbial biocatalysis. J Am Chem Soc 1999, 121:1603-1604.

16. Rodriguez A, Martinez JA, Baez-Viveros JL, Flores N, Hernandez-Chavez G, Ramirez OT, Gosset G, Bolivar F: Constitutive expression of selected genes from the pentose phosphate and aromatic pathways increases the shikimic acid yield in high-glucose batch cultures of an Escherichia coli strain lacking PTS and pykF. Microb Cell Fact 2013, 12:86

17. Iomantas YAV, Abalakina EG, Polanuer BM, Yampolskaya TA, Bachina TA, Kozlov YI: Method for producing shikimic acid. US patent 6436664 B1; 2002.

18. Sauer U, Hatzimanikatis V, Hohmann HP, Manneberg M, van Loon APGM Bailey JE: Physiology and metabolic fluxes of wild-type and riboflavinproducing Bacillus subtilis. Appl Environ Microb 1996, 62:3687-3696.

19. Davies WD, Davidson BE: The nucleotide sequence of aroG, the gene for 3-deoxy-D-arabinoheptulosonate-7-phosphate synthetase (phe) in Escherichia coli K12. Nucleic Acids Res 1982, 10:4045-4058.

20. Zurawski G, Gunsalus RP, Brown KD, Yanofsky C: Structure and regulation of aroH, the structural gene for the tryptophan-repressible 3-deoxy-Darabino-heptulosonic acid-7-phosphate synthetase of Escherichia coli. J Mol Biol 1981, 145:47-73.

21. Umbarger HE: Amino-acid biosynthesis and its regulation. Annu Rev Biochem 1978, 47:533-606.

22. Fry B, Zhu T, Domach MM, Koepsel RR, Phalakornkule C, Ataai MM: Characterization of growth and acid formation in a Bacillus subtilis pyruvate kinase mutant. Appl Environ Microb 2000, 66:4045-4049.

23. Sauer U, Eikmanns BJ: The PEP-pyruvate-oxaloacetate node as the switch point for carbon flux distribution in bacteria. FEMS Microbiol Rev 2005, 29:765-794

24. Zhu T, Pan Z, Domagalski N, Koepsel R, Ataai MM, Domach MM: Engineering of Bacillus subtilis for enhanced total synthesis of folic acid. Appl Environ Microb 2005, 71:7122-7129.

25. Nguyen HD, Nguyen QA, Ferreira RC, Ferreira LCS, Tran LT, Schumann W: Construction of plasmid-based expression vectors for Bacillus subtilis exhibiting full structural stability. Plasmid 2005, 54:241-248.

26. Anagnostopoulos C, Spizizen J: Requirements for Transformation in Bacillus subtilis. J Bacteriol 1961, 81:741-746.

27. Joseph P, Fantino JR, Herbaud ML, Denizot F: Rapid orientated cloning in a shuttle vector allowing modulated gene expression in Bacillus subtilis. FEMS Microbiol Lett 2001, 205:91-97.

28. Vagner $\mathrm{V}$, Dervyn $\mathrm{E}$, Ehrlich SD: A vector for systematic gene inactivation in Bacillus subtilis. Microbiology-Uk 1998, 144:3097-3104.

29. Fuhrer $\mathrm{T}$, Fischer $\mathrm{E}$, Sauer U: Experimental identification and quantification of glucose metabolism in seven bacterial species. J Bacteriol 2005, 187:1581-1590.

30. Nanchen A, Fuhrer T, Sauer U: Determination of metabolic flux ratios from ${ }^{13} \mathrm{C}$-experiments and gas chromatography-mass spectrometry data: protocol and principles. Methods Mol Biol 2007, 358:177-197.

31. Fischer E, Sauer U: Metabolic flux profiling of Escherichia coli mutants in central carbon metabolism using GC-MS. Eur J Biochem 2003, 270:880-891.

32. Yang C, Hua Q, Baba T, Mori H, Shimizu K: Analysis of Escherichia coli anaplerotic metabolism and its regulation mechanisms from the metabolic responses to altered dilution rates and phosphoenolpyruvate carboxykinase knockout. Biotechnol Bioeng 2003, 84:129-144.

33. Mollney M, Wiechert W, Kownatzki D, de Graaf AA: Bidirectional reaction steps in metabolic networks: IV. Optimal design of isotopomer labeling experiments. Biotechnol Bioeng 1999, 66:86-103.

doi:10.1186/1475-2859-13-40

Cite this article as: Liu et al: Metabolic flux responses to genetic modification for shikimic acid production by Bacillus subtilis strains. Microbial Cell Factories 2014 13:40.

\section{Submit your next manuscript to BioMed Central and take full advantage of:}

- Convenient online submission

- Thorough peer review

- No space constraints or color figure charges

- Immediate publication on acceptance

- Inclusion in PubMed, CAS, Scopus and Google Scholar

- Research which is freely available for redistribution

Submit your manuscript at www.biomedcentral.com/submit
C Biomed Central 